\title{
Direct measurement of the bulk spin structure of noncentrosymmetric BiTeCl
}

\author{
Gabriel Landolt, ${ }^{1,2}$ Sergey V. Eremeev, ${ }^{3,4,5}$ Oleg E. Tereshchenko, ${ }^{5,6,7}$ Stefan Muff,, ${ }^{2,8}$ Konstantin A. Kokh, ${ }^{5,7,9}$ \\ Jürg Osterwalder, ${ }^{1}$ Evgueni V. Chulkov, ${ }^{10}$ and J. Hugo Dil ${ }^{2,8}$ \\ ${ }^{1}$ Physik-Institut, Universität Zürich, Winterthurerstrasse 190, CH-8057 Zürich, Switzerland \\ ${ }^{2}$ Swiss Light Source, Paul Scherrer Institut, CH-5232 Villigen, Switzerland \\ ${ }^{3}$ Institute of Strength Physics and Materials Science, Russian Academy of Sciences, Siberian Branch, \\ Akademicheskiy prospekt 2/4, Tomsk 634021, Russia \\ ${ }^{4}$ Tomsk State University, Tomsk 634050, Russia \\ ${ }^{5}$ Saint Petersburg State University, Saint Petersburg 198504, Russia \\ ${ }^{6}$ Rzhanov Institute of Semiconductor Physics, Russian Academy of Sciences, Siberian Branch, \\ prospekt Akademika Lavrenteva 13, Novosibirsk 630090, Russia \\ ${ }^{7}$ Novosibirsk State University, Novosibirsk 636090, Russia \\ ${ }^{8}$ Institut de Physique de la Matière Condensée, Ecole Polytechnique Fédérale de Lausanne, CH-1015 Lausanne, Switzerland \\ ${ }^{9}$ Sobolev Institute of Geology and Mineralogy, Russian Academy of Sciences, Siberian Branch, \\ prospekt Koptyuga 3, Novosibirsk 630090, Russia \\ ${ }^{10}$ Donostia International Physics Center (DIPC) and CFM-MPC, Centro Mixto CSIC-UPV/EHU, \\ Departamento de Física de Materiales, UPV/EHU, E-20080 San Sebastián, Spain
}

(Received 5 September 2014; revised manuscript received 20 December 2014; published 10 February 2015)

\begin{abstract}
A strong spin-orbit interaction leads to a Rashba-type splitting of the bulk bands of $\mathrm{BiTeCl}$, which results in toroidal Fermi surfaces with distinct spin structures depending on the chemical potential. Here, we present spinand angle-resolved photoelectron spectroscopy measurements of the three-dimensional spin-orbit split state at the top of the valence band. Its polarization is systematically studied in dependence of the photon energy and compared to ab initio calculations of the initial state. The measured spin orientation is in plane to the (0001) surface and perpendicular to the momentum, independently of the photon energy.
\end{abstract}

In the aim of spintronics research to produce spin currents and manipulate spins by electric fields, the role of the spin-orbit interaction (SOI) is essential. Generally, the SOI significantly affects the band structure even in low- $Z$ materials (e.g., Ref. [1]). It is also responsible for the band inversion of topological insulators and the existence of their surface states [2]. SOI is the key mechanism of spin polarization in the absence of magnetism and causes the helical spin structure of topological surface states (TSSs) [3-5] and Rashba-split states in inversion asymmetric systems [6]. In nonequilibrium conditions the SOI allows for spin control as in the spin field effect transistor [7], or as in the case of SOI-induced spin torque exerted on local magnetic moments $[8,9]$.

Recently, the material class of semiconducting BiTe $X(X=$ $\mathrm{Cl}, \mathrm{Br}, \mathrm{I})$ came into focus $[10,11]$, because of a sizable spin splitting in the surface and bulk states [12-15], arising from a strong SOI and the materials' polar and noncentrosymmetric crystal structure [16]. The charge polarity and helicity of the spin structure is given by the stacking order of the atomic layers. A similar spin splitting is predicted in the bulk states of $\mathrm{GeTe}$, a ferroelectric semiconductor, where the spin orientation is expected to be switchable by reversing the spontaneous charge polarization [17].

In this Rapid Communication, we study the spin polarization of the bulk valence band (VB) of BiTeCl by means of spinand angle-resolved photoelectron spectroscopy (SARPES and ARPES). While the spin structures of two-dimensional surface or quantum well states have been extensively studied in the past [18], SARPES measurements of Rashba-split bulk bands are still lacking. We would like to emphasize that neither in this study, nor in our previous studies [19], did we find any evidence of topologically nontrivial states as recently claimed by Chen and co-workers [20].

The crystals were grown as described in Ref. [19] and structurally analyzed by x-ray diffraction measurements. All the ARPES experiments were performed at the COPHEE endstation of the Surface and Interface Spectroscopy beamline at the Swiss Light Source [21]. The in situ sample cleaving and the subsequent measurements were carried out at a sample temperature of $20 \mathrm{~K}$ and a base pressure of lower than $3 \times 10^{-10}$ mbar. The ARPES experiments were performed with an Omicron EA125 analyzer, and the spin information was obtained by a pair of orthogonally mounted $40 \mathrm{kV}$ classical Mott detectors, except for the measurements in Fig. 2, which were obtained in single Mott mode. Figure 1(a) schematically illustrates the experimental setup; the incoming $p$-polarized beam of light and the lens of the hemispherical analyzer form a $45^{\circ}$ angle. Angular photoelectron spectroscopy scans in the scattering plane or perpendicular to it are performed by rotating the sample about the $y$ or the $x$ axis, respectively.

The electronic structure calculations were performed within the density functional theory (DFT) as implemented in the Vienna $a b$ initio simulation package [22]. The all-electron projector augmented wave (PAW) [23] basis sets were used with the generalized gradient approximation (GGA) of Perdew, Burke, and Ernzerhof (PBE) to the exchange correlation (XC) potential. Relativistic corrections, including SOI, were taken into account.

The unit cell of $\mathrm{BiTeCl}$ (space group $C_{6 v}^{4}$ ) is built up out of two triple layers of alternating and hexagonally arranged $\mathrm{Bi}, \mathrm{Te}$, and $\mathrm{Cl}$ layers [Figs. 1(b) and 1(c)] [16]. The two triple layers are connected by a screw operation, a rotation 
(a)

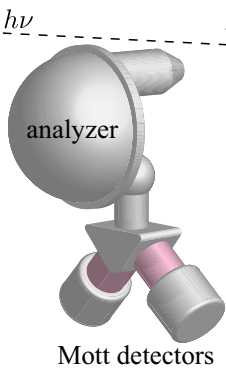

(c)

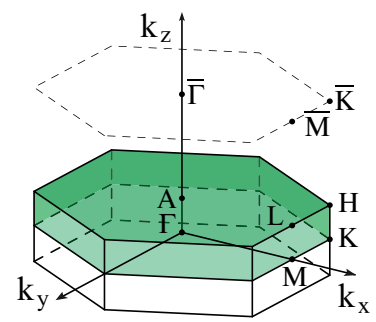

(e)
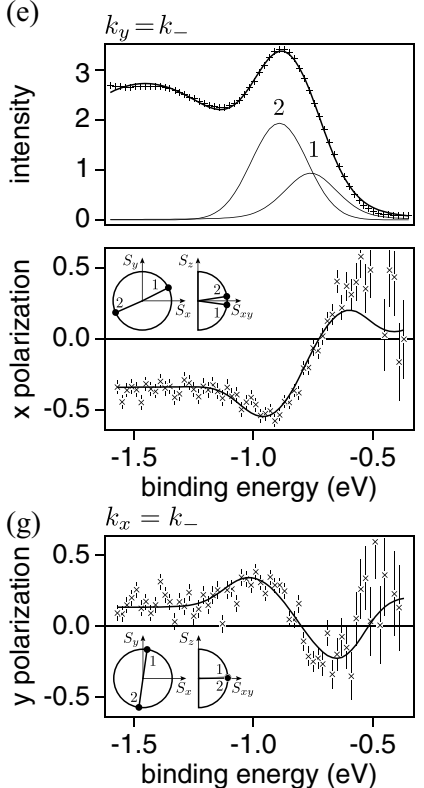

(b)

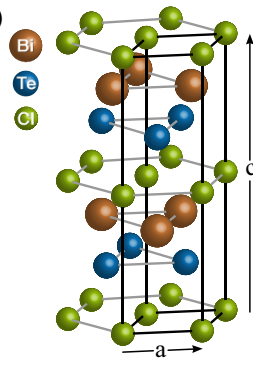

(d)

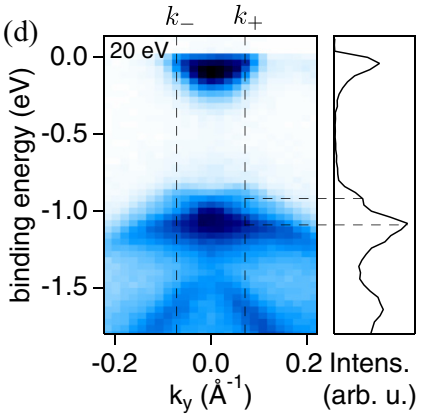

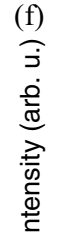

$k_{y}=k_{+}$
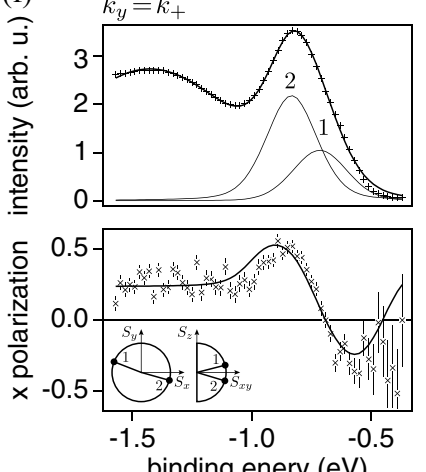

(h)

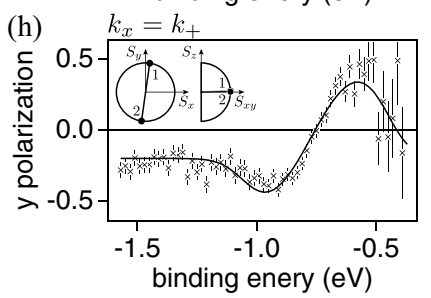

FIG. 1. (Color online) (a) Schematic illustration of the ARPES measurement geometry; the beam of light and the hemispherical analyzer form a $45^{\circ}$ angle. (b) $\mathrm{BiTeCl}$ crystal structure; the unit cell is marked with black lines. (c) $\mathrm{BiTeCl}$ hexagonal Brillouin zone. (d) Left panel: ARPES band map around the bulk $\Gamma$ point $(h v=20 \mathrm{eV})$ of Cl-terminated $\mathrm{BiTeCl}$. Right panel: EDC at the momentum of the $\operatorname{VBM}\left(k_{+} \approx 0.07 \AA^{-1}\right)$. (e) Spin-resolved EDC at $k_{y}=k_{-}=-k_{+}$. Top panel: Total intensity measured with the Mott detectors $(+)$. The lines represent the fitted total intensity and the energy-split top VB, respectively. Bottom panel: In-plane tangential polarization $S_{x}(\times)$ with the fitted curve (line). The inset shows a vectorial representation of the spin polarization of the individual bands. (f) Same as (e), but measured at $k_{+}$along $k_{y}$. (g) and (h) show the in-plane tangential polarization $\left(S_{y}\right)$ measured at $k_{x}=k_{-}$and $k_{+}$, respectively, confirming the helical spin texture.

by $60^{\circ}$, and a shift by half of the lattice parameter $c$ along the hexagonal axis. Space groups containing symmetry operations that are not a product of a point group operation and a lattice

translation, such as screw axes and glide plane operations, are called nonsymmorphic [24] and experimentally manifest in the photoemission selection rules [25,26]. In the case of $\mathrm{BiTeCl}$, the spectral intensity is suppressed around every other $\Gamma$ point in the out-of-plane momentum $\left(k_{z}\right)$ direction as a consequence of its screw axis [19].

The natural cleaving plane of $\mathrm{BiTeCl}$ lies between the $\mathrm{Te}$ and the $\mathrm{Cl}$ layers, such that the (0001) surface is terminated either by a pure $\mathrm{Te}$ or $\mathrm{Cl}$ atomic layer. While at Te-terminated (0001) BiTeCl surfaces several sets of surface states both in the VB and conduction band (CB) can be spectroscopically observed, no evidence of their theoretically anticipated counterparts on Cl-terminated surfaces can be found. Their absence is likely to be related to a photon-induced surface chemical effect [19]. The band map in the left panel of Fig. 1(d) shows the top of the VB and bottom of the $\mathrm{CB}$ of the bulk measured at a Cl-terminated surface with $20 \mathrm{eV}$ photon energy, roughly corresponding to the bulk $\Gamma K$ direction. The Rashba-type splitting of the top of the VB has its maximum at $k_{+}=-k_{-} \approx 0.07 \AA^{-1}$ and is around $170 \mathrm{meV}$, as shown in the energy distribution curve (EDC) in the right panel.

The SARPES experiments were performed at two momenta located symmetrically on either side of $\bar{\Gamma}$. Figures $1(\mathrm{e})$ and $1(\mathrm{f})$ show spin-resolved EDCs at $20 \mathrm{eV}$ photon energy across the top $\mathrm{VB}$ at $k_{-}$and $k_{+}$along the bulk $\Gamma K$ crystal direction, in this case the $y$ direction of the manipulator. While the top panels show the sum of the intensity profiles measured by each Mott channel, the bottom panels contain the measured photoelectron spin polarization in the direction tangential $\left(S_{x}\right)$ to the constant energy contour of the initial state. The radial and out-of-plane component of the spin polarization are small compared to the tangential component [27]. By simultaneously fitting the measured total intensity and the three measured spin polarization curves, a spin polarization vector is assigned to each band [28]. The numbered pseudo Voigt profiles in the top panels are the result of the fitting procedure. Their splitting is determined to be $(130 \pm 10) \mathrm{meV}$ and matches reasonably well with the spin-integrated measurement considering the larger angular integration range of a spin-resolved measurement. A representation of the spin polarization vectors of the individual bands can be found in the inset of the bottom panel.

For measurements with momenta along the $x$ direction of the manipulator, the spin polarization retains its mostly tangential $\left(S_{y}\right)$ orientation. Figures $1(\mathrm{~g})$ and $1(\mathrm{~h})$ show the tangential component of the spin polarization for $k_{x}=k_{-}$and $k_{+}$, respectively, measured along the bulk $\Gamma M$ crystal direction (see Ref. [27] for the radial and out-of-plane components). The insets show the result of the fitting procedure; within experimental resolution, no out-of-plane polarization is measured and the radial polarization is small.

The absence of surface states on Cl-terminated $\mathrm{BiTeCl}$ (0001) and the large spin-orbit coupling give one the opportunity to examine the evolution of the photoelectron polarization of a $k_{z}$ dispersing bulk state. The measured spin polarization is significantly reduced at higher photon energies of 22 and $24 \mathrm{eV}$, which roughly correspond to $k_{z}$ momenta halfway along the $\Gamma A$ line, and at the $A$ point, respectively [27]. Further, no reorientation away from its tangential orientation can be observed. At these low photon energies the $k_{z}$ broadening 

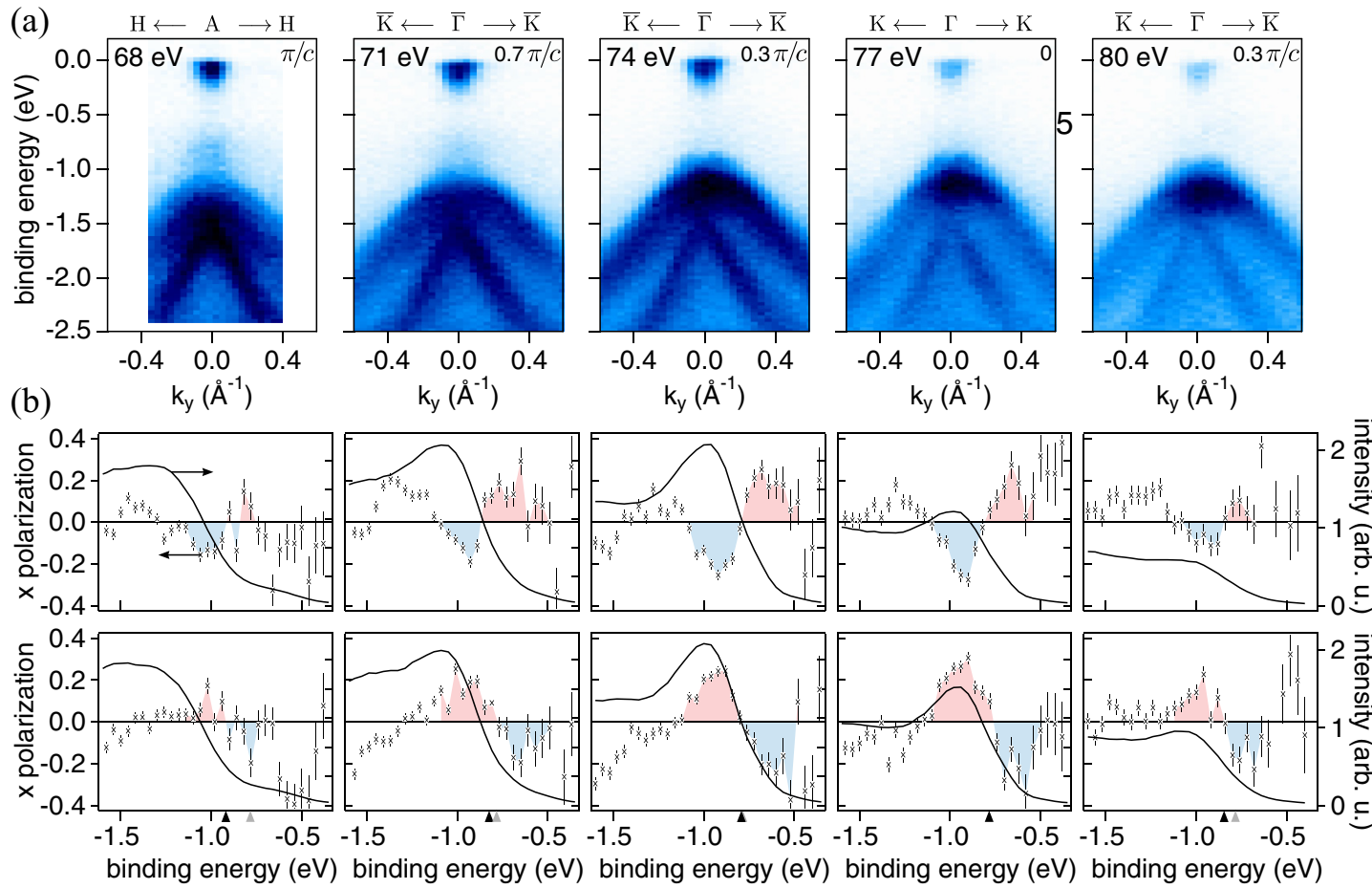

binding energy $(\mathrm{eV})$ binding energy $(\mathrm{eV})$ binding energy $(\mathrm{eV})$ binding energy $(\mathrm{eV})$ binding energy $(\mathrm{eV})$

FIG. 2. (Color online) (a) Band maps at different photon energies; 68 and $77 \mathrm{eV}$ photon energy roughly correspond to the $k_{z}$ of the $A$ and the $\Gamma$ point, respectively. (b) Spin-resolved EDCs at $k_{y} \approx \pm 0.11 \AA^{-1}$ at the same photon energies as in (a). The black triangles in the abscissa mark the energy of the zero crossing of the spin polarization averaged for both $k_{y}$ values; for reference, the zero crossing at the $\Gamma$ point is indicated by gray triangles.

is large due to the low probing depth [29], and half of the relatively small Brillouin zone is swept over roughly within an only $4 \mathrm{eV}$ wide photon energy window. In general, the $k_{z}$ resolution in ARPES is enhanced by increasing the photon energy, therefore we additionally measured photonenergy-dependent spin-resolved EDCs in the Brillouin zone around the ninth bulk $\Gamma$ point at roughly $77 \mathrm{eV}$ photon energy where the photoemission intensity is still sufficient for spin-resolved measurements. The spin-integrated band maps in Fig. 2(a) measured along the projected $\overline{\Gamma K}$ direction depict the dispersion between the $A^{8}$ point $(68 \mathrm{eV})$ and the $\Gamma^{9}$ point (77 eV).

The spin-resolved EDCs in Fig. 2(b) were performed at $k_{y} \approx \pm 0.11 \AA^{-1}$ on either side of normal emission along the $\overline{\Gamma K}$ crystal direction. The measurements were recorded starting with low photon energies with a total acquisition time of $14 \mathrm{~h}$. A progressive degradation of the sample surface is reflected in the diminishing intensity (solid lines) towards higher photon energies. The in-plane spin polarization $(x)$ of the top $\mathrm{VB}$ at $77 \mathrm{eV}$ is compatible with the corresponding measurement at $20 \mathrm{eV}$ photon energy, in the sense that the helicity is the same, while the maximally measured spin polarization is reduced by roughly $50 \%$. The mainly in-plane polarization is again accompanied by a small out-of-plane component [27]. Towards lower photon energies, i.e., closer towards the bulk $A$ point, the spin polarization amplitude gradually decreases. This increase towards the bulk $\Gamma$ point is observed contrary to the trend of decreasing sample quality with time and is compatible to the measurements at lower photon energies.
In order to understand the spin structure of the initial state, we performed fully relativistic DFT calculations as shown in Figs. 3(a)-3(d). The color of the data points [defined in the inset of Fig. 3(a)] reflects the degree of spin polarization, projected in plane and tangentially to the momentum vector. Figures 3(a)-3(c) show the in-plane dispersion and spin polarization of the VBs close to the band gap at (a) $k_{z}=0$ (along $K \Gamma M$ ), (b) $\frac{1}{2} \pi / c$ (along $\bar{K} \bar{\Gamma} M$ ), and (c) $\pi / c$ (along $H A L)$. The vertical dashed lines indicate the momentum of the valence band maximum (VBM).

Generally, the mirror symmetry of the crystal demands the spin polarization to be fully in plane and tangential along $\bar{\Gamma} M$ for any $k_{z}$. While out-of-plane spin polarization is allowed for, e.g., the related compound $\mathrm{BiTeI}$ along and around the $\overline{\Gamma K}$ crystal direction [10], in the case of $\mathrm{BiTeCl}$, the screw operation symmetry inhibits out-of-plane spin polarization of any band in the entire Brillouin zone. Figure 3(d) shows the $k_{z}$ dispersion of the same bands at fixed in-plane momenta $k=$ $k_{+}(\overline{\Gamma \bar{M}}), 0(\bar{\Gamma})$, and $k_{-}(\overline{\Gamma \bar{K}})$, corresponding to the momenta of the VBM on either side of the $\bar{\Gamma}$ point and the zone center, respectively. Along the entire $\Gamma A$ line all the bands are doubly spin degenerate. Compared to $\mathrm{BiTeI}$, in $\mathrm{BiTeCl}$ the unit cell is doubled, which leads to a band backfolding about the $H A L$ plane and a doubling of the number of bands. Within the $H A L$ plane, the screw symmetry prohibits band gaps and the bands are at least doubly degenerate while retaining a finite spin polarization [24-26]. In order for two bands with the same spin polarization to meet in the $H A L$ plane, the band order is interchanged at a $k_{z}$ close to the zone boundary: In the $\overline{\Gamma K}$ direction, two bands of opposite spin polarization hybridize and swap the 
(a)
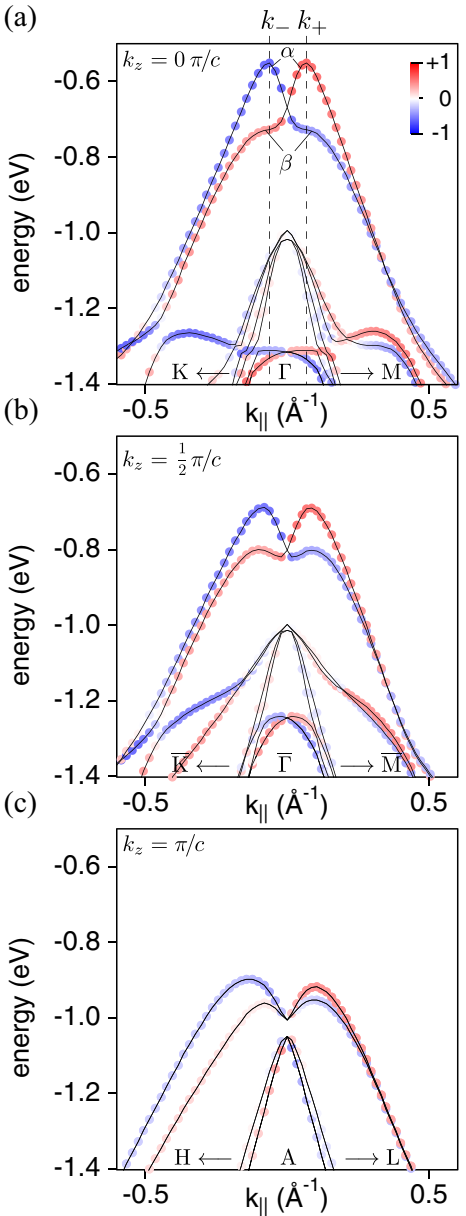

(d)
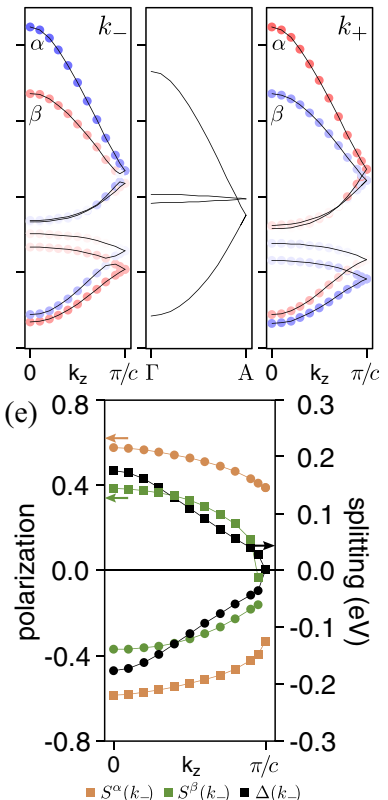

(f) $S^{\alpha}\left(k_{+}\right) \bullet S^{\beta}\left(k_{+}\right) \bullet \Delta\left(k_{+}\right)$

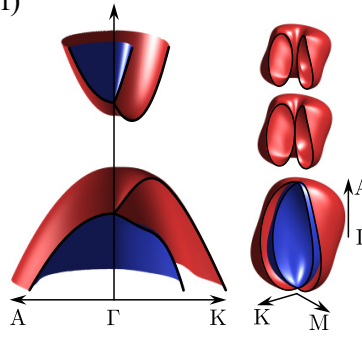

FIG. 3. (Color online) Relativistic DFT calculations of the $\mathrm{BiTeCl} \mathrm{VB}$ dispersion and spin structure close to the bulk band gap along $\overline{K \Gamma M}$ at (a) $k_{z}=0$, (b) $k_{z}=\frac{1}{2} \pi / c$, and (c) $k_{z}=\pi / c$. The color of the data points indicates the degree of polarization, which is purely in plane and perpendicular to the in-plane momentum. The dashed lines indicate the VBM at $k_{\|}=k_{-}$and $k_{+}$. (d) $k_{z}$ dispersion and spin structure for $k_{\|}=k_{-}$(left panel), 0 (center panel), and $k_{-}$(right panel). (e) $k_{z}$ dependence of the spin polarization for the topmost VBs $\alpha$ and $\beta S_{\alpha, \beta}(k)$. Also shown is the energy splitting between $\alpha$ and $\beta[\Delta(k)]$. (f) Left: Schematic illustration of the dispersion. Right: Transition of the Fermi surface topology depending on the chemical potential. The colors indicate the helicity of the spin structure.

sign of their polarization [left panel of Fig. 3(d)], whereas in the $\bar{\Gamma} M$ direction they cross each other [right panel of Fig. 3(d)].

Apart from the momentum region where the mentioned hybridization takes place, the spin polarization is gradually reduced towards the $H A L$ zone boundary plane, as depicted in Fig. 3(e). The graph, on the one hand, shows the $k_{z}$ dependence of the tangential spin polarization of the energy-split top band [ $\alpha$ and $\beta$, cf. Fig. 3(a)] at the in-plane momenta of the VBM along $\overline{\Gamma K}\left[S^{\alpha}\left(k_{-}\right)\right.$and $\left.S^{\beta}\left(k_{-}\right)\right]$and along $\overline{\Gamma M}\left[S^{\alpha}\left(k_{+}\right)\right.$and $S^{\beta}\left(k_{+}\right)$]. In addition, the energy splitting of the top VB steadily decreases towards the $A$ point $\left[\Delta\left(k_{-}\right)\right.$and $\left.\Delta\left(k_{+}\right)\right]$. Based on the theoretical calculations, a schematic illustration of the initial state Fermi surface of $\mathrm{BiTeCl}$ and its in-plane helical spin structure is given in Fig. 3(f). Note that DFT calculations only model the spin polarization of the initial state; however,

extensive photoemission calculations including the final states are beyond the scope of this experimental work.

It has been shown that circular dichroism (CD) measurements of the surface states of $\mathrm{BiTeCl}$ and related compounds are strongly photon energy dependent and thus CD is not a suitable probe for the spin polarization [30,31]. It is under debate how and whether the photoelectron spin polarization measured in SARPES portrays the spin structure of the initial state. The role of orbital selectivity of the photoemission process seems crucial, since the different orbitals forming an initial state couple to distinct spin structures [32,33]. Several mechanisms that additionally introduce photon energy dependence into the spin signal are also under investigation for either certain energy ranges [34] or generally [35] due to interference of the layer-dependent spin-orbital texture.

The above-mentioned studies refer to two-dimensional states. In three dimensions the optical selectivity rules apply similarly; in the used geometry, the spin signal of the top of the VB derives mostly from the dominant orbitals with $p_{z}$ character. Because of the finite probing depth of ARPES and the truncation of the crystal, spin-dependent interference effects can in principle also occur in bulk states [36]. The SARPES data of three-dimensional states presented in this Rapid Communication, however, show a photoelectron spin polarization that, for the used photon energies, is consistent with the DFT calculation of the initial states; most importantly, the helical rotation tangentially to the in-plane constant energy contour is independent of the experimental geometry [cf. Figs. 1(e)-1(h)]. The photon-energy-dependent reduction of the maximally measured degree of spin polarization towards the zone boundary can be explained with the reducing energy splitting and the band crossing on either side of the $H A L$ plane. In addition, the steep $k_{z}$ dispersion of the initial states around the $H A L$ zone boundary leads to a decreased resolution due to the intrinsic energy and $k_{z}$ broadening [29]. The $k_{z}$ broadening at the used photon energies is in the order of $20 \%$ of the Brillouin zone $\left(2 \pi / c \approx 0.51 \AA^{-1}\right.$ [16]). SARPES measurements in the soft $\mathrm{x}$-ray photon energy regime which would significantly increase the $k_{z}$ resolution are not feasible to date.

In summary, we have performed systematic spin-resolved measurements on the bulk $\mathrm{VB}$ of $\mathrm{BiTeCl}$ at various photon energies corresponding to different $k_{z}$ in the bulk Brillouin zone. Using $p$-polarized light we find no reorientation of the spin polarization vector within the studied photon energy ranges. We did observe a significant reduction of the degree of spin polarization in the $k_{z}$ direction towards the Brillouin zone boundary, where the bands are doubly degenerate but still spin polarized. The reduction of the measured spin polarization can be explained by an increased band overlap and limited $k_{z}$ resolution. Our data not only help in the understanding of the spin-polarized bands of $\mathrm{BiTeCl}$ and the related $\mathrm{BiTe} X$ materials but contribute to the discussion about the mechanism of spin polarization in ARPES.

We acknowledge partial support from the Basque Country Government, Departamento de Educación, Universidades e Investigación (Grant No. IT-756-13), the Spanish Ministerio de Ciencia e Innovación (Grant No. FIS2010-19609-C02-01), 
the Ministry of Education and Science of the Russian Federation (Grant No. 2.8575.2013), the Russian Foundation for Basic Research (Grants No. 15-02-02717, No. 15-02-
01797), and the Saint Petersburg State University (Project No. 11.50.202.2015). This work was supported by the Swiss National Science Foundation Project No. PP00P2 144742/1.
[1] J. R. Chelikowsky and M. L. Cohen, Nonlocal pseudopotential calculations for the electronic structure of eleven diamond and zinc-blende semiconductors, Phys. Rev. B 14, 556 (1976).

[2] L. Fu, C. L. Kane, and E. J. Mele, Topological insulators in three dimensions, Phys. Rev. Lett. 98, 106803 (2007).

[3] C. L. Kane and E. J. Mele, $Z_{2}$ topological order and the quantum spin Hall effect, Phys. Rev. Lett. 95, 146802 (2005).

[4] M. König, S. Wiedmann, C. Brüne, A. Roth, H. Buhmann, L. W. Molenkamp, X.-L. Qi, and S.-C. Zhang, Quantum spin hall insulator state in $\mathrm{HgTe}$ quantum wells, Science 318, 766 (2007).

[5] D. Hsieh, Y. Xia, D. Qian, L. Wray, J. Dil, F. Meier, J. Osterwalder, L. Patthey, J. Checkelsky, N. Ong, A. Fedorov, H. Lin, A. Bansil, D. Grauer, Y. Hor, R. Cava, and M. Hasan, A tuneable topological insulator in the spin helical Dirac transport regime, Nature (London) 460, 1101 (2009).

[6] Y. A. Bychkov and E. I. Rashba, Properties of a 2D electron gas with lifted spectral degeneracy, JETP Lett. 39, 78 (1984).

[7] S. Datta and B. Das, Electronic analog of the electro-optic modulator, Appl. Phys. Lett. 56, 665 (1990).

[8] A. Manchon and S. Zhang, Theory of spin torque due to spinorbit coupling, Phys. Rev. B 79, 094422 (2009).

[9] I. Mihai Miron, G. Gaudin, S. Auffret, B. Rodmacq, A. Schuhl, S. Pizzini, J. Vogel, and P. Gambardella, Current-driven spin torque induced by the Rashba effect in a ferromagnetic metal layer, Nat. Mater. 9, 230 (2010).

[10] K. Ishizaka, M. S. Bahramy, H. Murakawa, M. Sakano, T. Shimojima, T. Sonobe, K. Koizumi, S. Shin, H. Miyahara, A. Kimura, K. Miyamoto, T. Okuda, H. Namatame, M. Taniguchi, R. Arita, N. Nagaosa, K. Kobayashi, Y. Murakami, R. Kumai, Y. Kaneko, Y. Onose, and Y. Tokura, Giant Rashba-type spin splitting in bulk BiTeI, Nat. Mater. 10, 521 (2011).

[11] S. V. Eremeev, I. A. Nechaev, Y. M. Koroteev, P. M. Echenique, and E. V. Chulkov, Ideal two-dimensional electron systems with a giant Rashba-type spin splitting in real materials: Surfaces of bismuth tellurohalides, Phys. Rev. Lett. 108, 246802 (2012).

[12] G. Landolt, S. V. Eremeev, Y. M. Koroteev, B. Slomski, S. Muff, T. Neupert, M. Kobayashi, V. N. Strocov, T. Schmitt, Z. S. Aliev, M. B. Babanly, I. R. Amiraslanov, E. V. Chulkov, J. Osterwalder, and J. H. Dil, Disentanglement of surface and bulk Rashba spin splittings in noncentrosymmetric BiTeI, Phys. Rev. Lett. 109, 116403 (2012).

[13] A. Crepaldi, L. Moreschini, G. Autès, C. Tournier-Colletta, S. Moser, N. Virk, H. Berger, P. Bugnon, Y. J. Chang, K. Kern, A. Bostwick, E. Rotenberg, O. V. Yazyev, and M. Grioni, Giant ambipolar Rashba effect in the semiconductor BiTeI, Phys. Rev. Lett. 109, 096803 (2012)

[14] M. Sakano, M. S. Bahramy, A. Katayama, T. Shimojima, H. Murakawa, Y. Kaneko, W. Malaeb, S. Shin, K. Ono, H. Kumigashira, R. Arita, N. Nagaosa, H. Y. Hwang, Y. Tokura, and K. Ishizaka, Strongly spin-orbit coupled two-dimensional electron gas emerging near the surface of polar semiconductors, Phys. Rev. Lett. 110, 107204 (2013).

[15] I. P. Rusinov, I. A. Nechaev, S. V. Eremeev, C. Friedrich, S. Blügel, and E. V. Chulkov, Many-body effects on the rashba-type spin splitting in bulk bismuth tellurohalides, Phys. Rev. B 87, 205103 (2013).

[16] A. Shevelkov, E. Dikarev, R. Shpanchenko, and B. Popovkin, Crystal structures of bismuth tellurohalides BiTeX $(\mathrm{X}=\mathrm{Cl}, \mathrm{Br}$, I) from X-ray powder diffraction data, J. Solid State Chem. 114, 379 (1995).

[17] D. Di Sante, P. Barone, R. Bertacco, and S. Picozzi, Electric control of the giant Rashba effect in bulk GeTe, Adv. Mater. 25 , 509 (2013).

[18] J. H. Dil, Spin and angle resolved photoemission on nonmagnetic low-dimensional systems, J. Phys.: Condens. Matter 21, 403001 (2009).

[19] G. Landolt, S. V. Eremeev, O. E. Tereshchenko, S. Muff, B. Slomski, K. A. Kokh, M. Kobayashi, T. Schmitt, V. N. Strocov, J. Osterwalder, E. V. Chulkov, and J. H. Dil, Bulk and surface rashba splitting in single termination $\mathrm{BiTeCl}$, New J. Phys. 15, 085022 (2013).

[20] Y. L. Chen, M. Kanou, Z. K. Liu, H. J. Zhang, J. A. Sobota, D. Leuenberger, S. K. Mo, B. Zhou, S.-L. Yang, P. S. Kirchmann, D. H. Lu, R. G. Moore, Z. Hussain, Z. X. Shen, X. L. Qi, and T. Sasagawa, Discovery of a single topological Dirac fermion in the strong inversion asymmetric compound $\mathrm{BiTeCl}$, Nat. Phys. 9, 704 (2013).

[21] M. Hoesch, T. Greber, V. N. Petrov, M. Muntwiler, M. Hengsberger, W. Auwaerter, and J. Osterwalder, Spinpolarized Fermi surface mapping, J. Electron Spectrosc. Relat. Phenom. 124, 263 (2002).

[22] G. Kresse and J. Hafner, Ab initio molecular dynamics for liquid metals, Phys. Rev. B 47, 558 (1993).

[23] G. Kresse and D. Joubert, From ultrasoft pseudopotentials to the projector augmented-wave method, Phys. Rev. B 59, 1758 (1999).

[24] M. Dresselhaus, G. Dresselhaus, and A. Jorio, Group Theory (Springer, Berlin, 2008).

[25] D. Pescia, A. Law, M. Johnson, and H. Hughes, Determination of observable conduction band symmetry in angle-resolved electron spectroscopies: Non-symmorphic space groups, Solid State Commun. 56, 809 (1985).

[26] T. Finteis, M. Hengsberger, T. Straub, K. Fauth, R. Claessen, P. Auer, P. Steiner, S. Hüfner, P. Blaha, M. Vögt, M. LuxSteiner, and E. Bucher, Occupied and unoccupied electronic band structure of $\mathrm{WSe}_{2}$, Phys. Rev. B 55, 10400 (1997).

[27] See Supplemental Material at http://link.aps.org/supplemental/ 10.1103/PhysRevB.91.081201 for additional spin-resolved EDCs.

[28] F. Meier, H. Dil, J. Lobo-Checa, L. Patthey, and J. Osterwalder, Quantitative vectorial spin analysis in angle-resolved photoemission: $\mathrm{Bi} / \mathrm{Ag}(111)$ and $\mathrm{Pb} / \mathrm{Ag}(111)$, Phys. Rev. B 77, 165431 (2008). 
[29] V. Strocov, Intrinsic accuracy in 3-dimensional photoemission band mapping, J. Electron Spectrosc. Relat. Phenom. 130, 65 (2003).

[30] A. Crepaldi, F. Cilento, M. Zacchigna, M. Zonno, J. C. Johannsen, C. Tournier-Colletta, L. Moreschini, I. Vobornik, F. Bondino, E. Magnano, H. Berger, A. Magrez, P. Bugnon, G. Autès, O. V. Yazyev, M. Grioni, and F. Parmigiani, Momentum and photon energy dependence of the circular dichroic photoemission in the bulk Rashba semiconductors BiTeX (X = I, Br, Cl), Phys. Rev. B 89, 125408 (2014).

[31] M. R. Scholz, J. Sánchez-Barriga, J. Braun, D. Marchenko, A. Varykhalov, M. Lindroos, Y. J. Wang, H. Lin, A. Bansil, J. Minár, H. Ebert, A. Volykhov, L. V. Yashina, and O. Rader, Reversal of the circular dichroism in angle-resolved photoemission from $\mathrm{Bi}_{2} \mathrm{Te}_{3}$, Phys. Rev. Lett. 110, 216801 (2013).

[32] H. Zhang, C.-X. Liu, and S.-C. Zhang, Spin-orbital texture in topological insulators, Phys. Rev. Lett. 111, 066801 (2013).

[33] Y. Cao, J. A. Waugh, X.-W. Zhang, J.-W. Luo, Q. Wang, T. J. Reber, S. K. Mo, Z. Xu, A. Yang, J. Schneeloch, G. D. Gu,
M. Brahlek, N. Bansal, S. Oh, A. Zunger, and D. S. Dessau, Mapping the orbital wavefunction of the surface states in threedimensional topological insulators, Nat. Phys. 9, 499 (2013).

[34] J. Sanchez-Barriga, A. Varykhalov, J. Braun, S.-Y. Xu, N. Alidoust, O. Kornilov, J. Minar, K. Hummer, G. Springholz, G. Bauer, R. Schumann, L. V. Yashina, H. Ebert, M. Z. Hasan, and $\mathrm{O}$. Rader, Photoemission of $\mathrm{Bi}_{2} \mathrm{Se}_{3}$ with circularly polarized light: Probe of spin polarization or means for spin manipulation? Phys. Rev. X 4, 011046 (2014).

[35] Z. H. Zhu, C. N. Veenstra, G. Levy, A. Ubaldini, P. Syers, N. P. Butch, J. Paglione, M. W. Haverkort, I. S. Elfimov, and A. Damascelli, Layer-by-layer entangled spin-orbital texture of the topological surface state in $\mathrm{Bi}_{2} \mathrm{Se}_{3}$, Phys. Rev. Lett. 110, 216401 (2013).

[36] A. Kimura, E. E. Krasovskii, R. Nishimura, K. Miyamoto, T. Kadono, K. Kanomaru, E. V. Chulkov, G. Bihlmayer, K. Shimada, H. Namatame, and M. Taniguchi, Strong Rashbatype spin polarization of the photocurrent from bulk continuum states: Experiment and theory for Bi(111), Phys. Rev. Lett. 105, 076804 (2010). 